\title{
Effects of the dip angle in the induction anisotropy logs
}

Carvalho P. R. * (ICIBE/UFRA), Régis C. R. T. (CPGf/UFPA) and Silva V. S. (Castanhal/UFPA)

Copyright 2018, SBGf - Sociedade Brasileira de Geofísica

Este texto foi preparado para a apresentação no VIII Simpósio Brasileiro de Geofísica, Salinópolis, 18 a 20 de setembro de 2018. Seu conteúdo foi revisado pelo Comitê Técnico do VIII SimBGf mas não necessariamente representa a opinião da SBGf ou de seus associados. É proibida a reprodução total ou parcial deste material para propósitos comerciais sem prévia autorização da SBGf.

\section{Abstract}

Some of the most modern induction well logging tools provide resistivity anisotropy logs and estimates of the electrical resistivity of sand laminae in thinly laminated formations.

In this work, coaxial and coplanar logs were modeled in one-dimensional (1D) laminated packages, neglecting the presence of the borehole and the invasion zones, to simulate geological environments of hydrocarbon reservoirs with electrical anisotropy.

The objective of this paper is to perform an analysis of the effects of the well's inclination angle on the induction tool response in a thinly laminated reservoir. Thus, it's shown only the responses from coaxial and coplanar coil array, which are used to produce anisotropy logs as well as to estimate the sand conductivity of the formation.

The results show the strong sensitivity of the coaxial and coplanar signals to the deviated angle and, consequently, these angle's effects on the anisotropy logs.

\section{Introduction}

Current triaxial or multicomponent induction probes consist basically of a combination of a coaxial arrangement with two coplanar arrangements of coils (Figure 01), i.e., three sources and three sensors, with axes orthogonal to each other (Krigshäuser et al., 2000). These probes were designed originally to investigate thinly laminated reservoirs, with a structural anisotropic behavior. The responses of the various arrangements of coils are simultaneously registered on multiple channels at multiple frequencies and source-sensor spacing.

Currently, besides being the main location tool of finely laminated reservoirs, these triaxial sources and sensors are also applied in many situations of asymmetric geometry, such as locating dissolution cavities (vugs) and fractured zones in the vicinity of the wells, monitoring invasion fronts in horizontal wells, among others (Omeragic et al, 2015).

In this work, we show some numerical responses for onedimensional (1D) layered anisotropic geoelectric models, in which the presence of the borehole and the invasion zones are neglected, to simulate deviated logs with respect to bedding within geological environments of hydrocarbon reservoirs with structural electrical anisotropy.

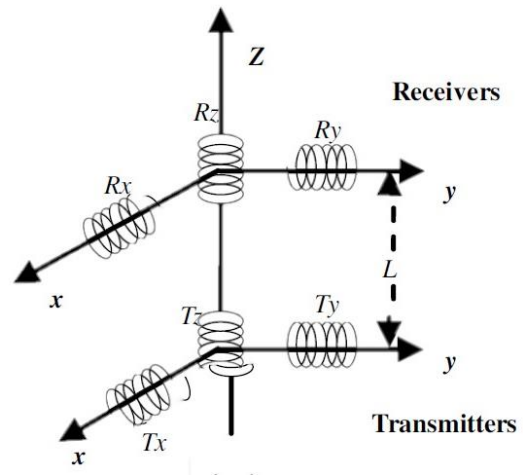

Figure 01 - Basic structure of the modern induction tools (Adapted from Moran \& Gianzero, 1979).

Our goals here are to investigate the effects of the dip angle in the induction tool response and to investigate how much the probe's inclination affects the apparent anisotropy logs obtained from the ratio of the coaxial to the coplanar responses.

\section{Method}

In the theoretical treatment, the coils are represented as point magnetic dipoles, as illustrated in Figure 02. The dip angle $\theta$ is determined by the orientation of the borehole (azimuth and deviation) and the orientation of the formation (dip and strike). This angle can be caused by deviated wells in flat formations, by vertical wells in dipping beds, or by any combination thereof.

The magnetic fields components from the vertical (VMD) and horizontal (HMD) magnetic dipoles are calculated using Schelkunoff potentials as described in Carvalho et al. (2010).

The semi-analytical responses of the 1D models result in improper integrals due to the inverse Hankel transform, which are solved numerically with a 21-point GaussKronrod quadrature rule.

The fields from an inclined dipole are calculated as a combination of those from a vertical $\left(H^{v}\right)$ and a horizontal $\left(H^{h}\right)$ source. Equations (1) and (2) yield the coaxial $\left(H^{c x}\right)$ and coplanar $\left(H^{c p}\right)$ responses in terms of the component of the magnetic field normal to their receivers:

$$
\begin{aligned}
& H^{c x}=\left(H_{z}^{v}+H_{z}^{h}\right) \cos \theta+\left(H_{x}^{v}+H_{x}^{h}\right) \sin \theta, \\
& H^{c p}=\left(H_{z}^{v}+H_{z}^{h}\right) \sin \theta+\left(H_{x}^{v}+H_{x}^{h}\right) \cos \theta .
\end{aligned}
$$




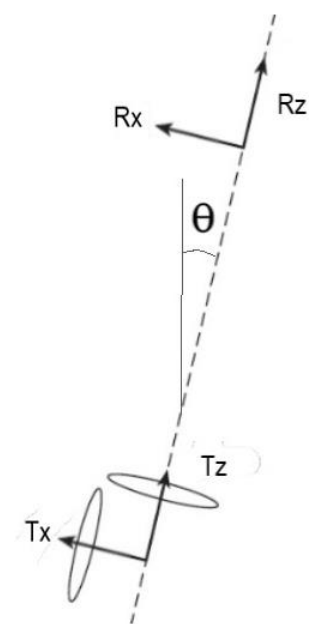

Figure 02 - The coaxial $\left(T_{z} / R_{z}\right)$ and coplanar $\left(T_{x} / R_{x}\right)$ equivalent model (Adapted from Ellis \& Singer, 2007).

The complex conductivity (Eqs. 3 and 4) may be written in terms of the registered magnetic fields as

$\sigma_{R}^{c x}+i \sigma_{X}^{c x}=\left(2 i / \omega \mu L^{2}\right) h^{c x}$,

$\sigma_{R}^{c p}+i \sigma_{X}^{c p}=\left(-2 i / \omega \mu L^{2}\right) h^{c p}$,

where $h^{c x}$ and $h^{c p}$ are the secondary magnetic fields which come exclusively from the medium, i.e., without the transmitter/receiver mutual coupling terms, and $L$ is the coil spacing.

The coaxial (Moran \& Kunz, 1962) and coplanar (Carvalho \& Verma, 1999) boosted signals with a partial correction of the Skin Effect are obtained by

$\sigma_{b}^{c x}=\sigma_{R}^{c x} /\left[1-(2 / 3)\left(L / \delta^{c x}\right)\right]$,

$\sigma_{b}^{c p}=\sigma_{R}^{c p} /\left[1-(4 / 3)\left(L / \delta^{c p}\right)\right]$,

where $\quad \delta^{c x}=\left[2 /\left(\omega \mu \sigma_{R}^{c x}\right)\right]^{1 / 2} \quad$ and $\quad \delta^{c p}=[2 /$ $\left.\left(\omega \mu \sigma_{R}^{c p}\right)\right]^{1 / 2}$.

When the source and receiver coil axes are both oriented perpendicular to the horizontal bedding planes, as in the case of the vertical coaxial array $(\theta=0)$, only the component of the conductivity parallel to the planes affects the response, i.e., horizontal conductivity $\sigma_{h}$. On the other hand, when both coils have axes oriented parallel to the bedding planes, as in the case of a vertical coplanar array, the vertical conductivity $\sigma_{v}$ affects the response significantly.

The vertical coaxial signal will be biased towards the high conductivity laminations (no oil-shale) because the circular induced currents flow parallel to the bedding planes, i.e., the anisotropy has no effect. However, elliptical induced currents from a vertical coplanar array will flow perpendicular to the borehole and bed interfaces, so that polarization charges accumulate at these boundaries. The coplanar signal is due to both horizontal $\left(\sigma_{h}\right)$ and vertical $\left(\sigma_{v}\right)$ conductivities, with $\sigma_{h}$ usually larger than $\sigma_{v}$ in the clastic sedimentary formations.
In Transversely Isotropic layers with a Vertical axis of symmetry (TIV), in which the main anisotropy directions are the same as the coordinate axes, the conductivity tensor reduces to

$\tilde{\sigma}=\left[\begin{array}{ccc}\sigma_{h} & 0 & 0 \\ 0 & \sigma_{h} & 0 \\ 0 & 0 & \sigma_{v}\end{array}\right]$.

This type of anisotropic medium has a characteristic parameter named coefficient of anisotropy, defined as

$$
\lambda^{2}=\sigma_{h} / \sigma_{v} \text {. }
$$

Kaufman \& Dashevsky (2010) deduced through current density distribution and Anderson et al. (2008) show through circuit theory (parallel and series resistors) an identical relation between the horizontal and vertical conductivities of the homogeneous anisotropic media and the conductivities of the thinly laminated medium formed by two alternating and distinct laminae $\left(\sigma_{s d}\right.$ and $\left.\sigma_{s h}\right)$ when their thicknesses are less than the tool's vertical resolution:

$\sigma_{h}=\sigma_{s d} V_{s d}+\sigma_{s h} V_{s h}$

$\sigma_{v}=\left(V_{s d} / \sigma_{s d}+V_{s h} / \sigma_{s h}\right)^{-1}$

where $V_{s d}$ and $V_{s h}$ are the volume fractions of each material which are obtained by spectroscopy probe. Thus, the sand laminae conductivity can be estimated from the horizontal $\left(\sigma_{h} \cong \sigma_{b}^{c x}\right)$ and vertical $\left(\sigma_{v} \cong \sigma_{b}^{c p}\right)$ conductivities (Eqs. 09 and 10) and, finally, its reciprocal value may be applied in Archie's equation to estimate the water saturation in the thinly laminated reservoir (Clavaud et al., 2005).

Kaufman \& Dashevsky (2003) show that in the low frequency range $(L / \delta \ll 1)$ the quadrature component of the secondary magnetic field (without the mutual term) registered by vertical coplanar array is directly proportional to the vertical conductivity $\left(\sigma_{v}\right)$. For this reason, measuring the ratio of the quadrature components of the coaxial and coplanar arrays allows us to obtain an apparent coefficient of anisotropy $\left(\lambda_{a}^{2}\right)$ which is the same as that given by the ratio of the boosted signals of the coplanar and coaxial arrays:

$$
\lambda^{2}=\sigma_{h} / \sigma_{v} \cong Q h_{x}^{c p} / Q h_{z}^{c x}={ }^{\sigma_{b}^{c p}} / \sigma_{b}^{c x}=\lambda_{a}^{2} .
$$

Anderson et al. (2008) show that this anisotropic index is a useful measurement for determining the level of anisotropy, and that when this ratio is higher than five, it alerts the log analyst to look for potential laminated-pay reservoir.

\section{Results}

The vertical coordinates in the figures are defined as follows: 1) "vertical depth" is the vertical distance from any 
on e point in the well to the horizontal bed center and 2) "measure depth" is the depth measured by logs along the borehole.
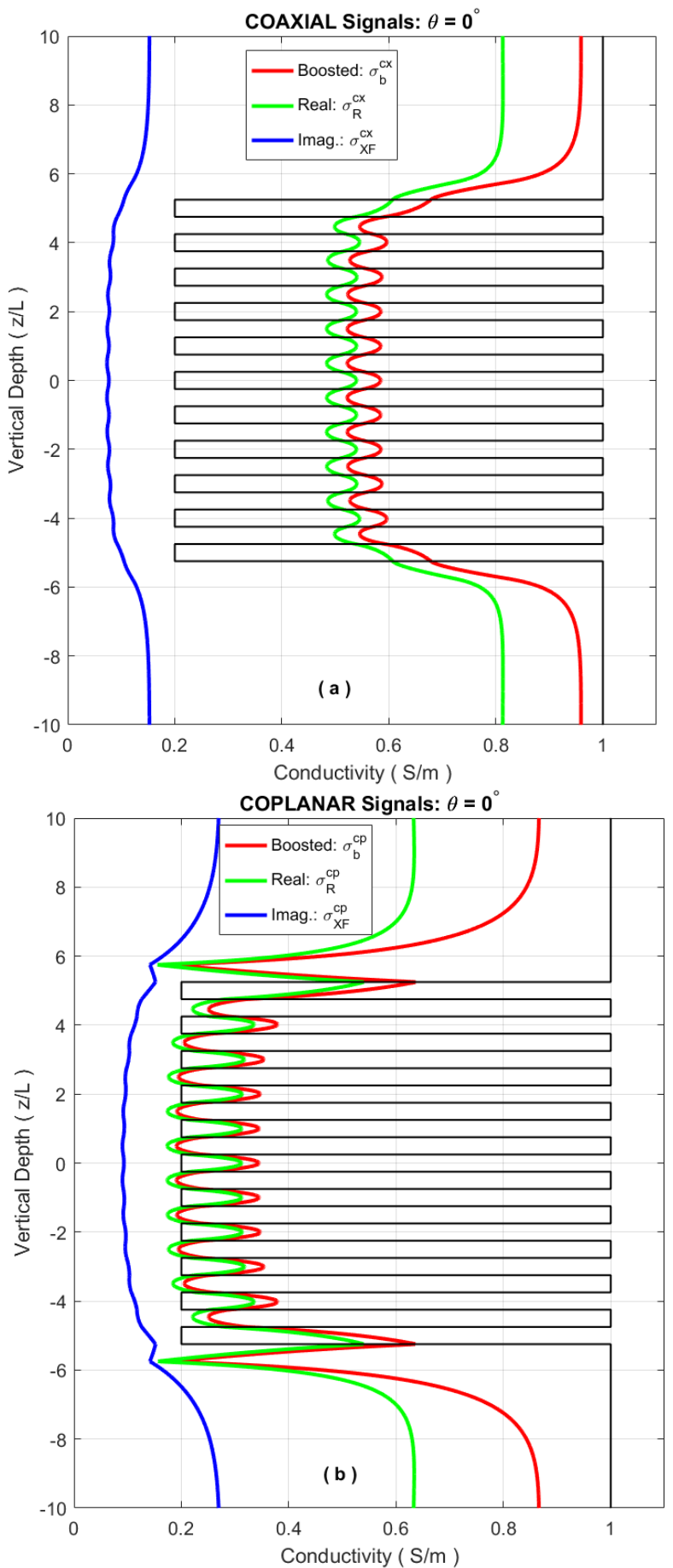

Figure 3 - Resistive, reactive and boosted of the (a) coaxial and (b) coplanar coil arrays to a thinly laminated formation $(h=L / 2)$.

Figure 3 shows the vertical coaxial (a) and coplanar (b) logs. This thick package $(10.5 \mathrm{~L})$ consists of a laminated
$(\mathrm{L} / 2)$ formation with a relative low conductivity contrast $\left(\sigma_{\mathrm{sd}}=0.2 \mathrm{~S} / \mathrm{m}\right.$ and $\left.\sigma_{\text {sh }}=1.0 \mathrm{~S} / \mathrm{m}\right)$.

The coplanar signals have a more prominent oscillation in the package although they suffer the strongest adjacent bed and skin effects. Polarizations "horns" appear in the coplanar profiles, against the package boundaries.
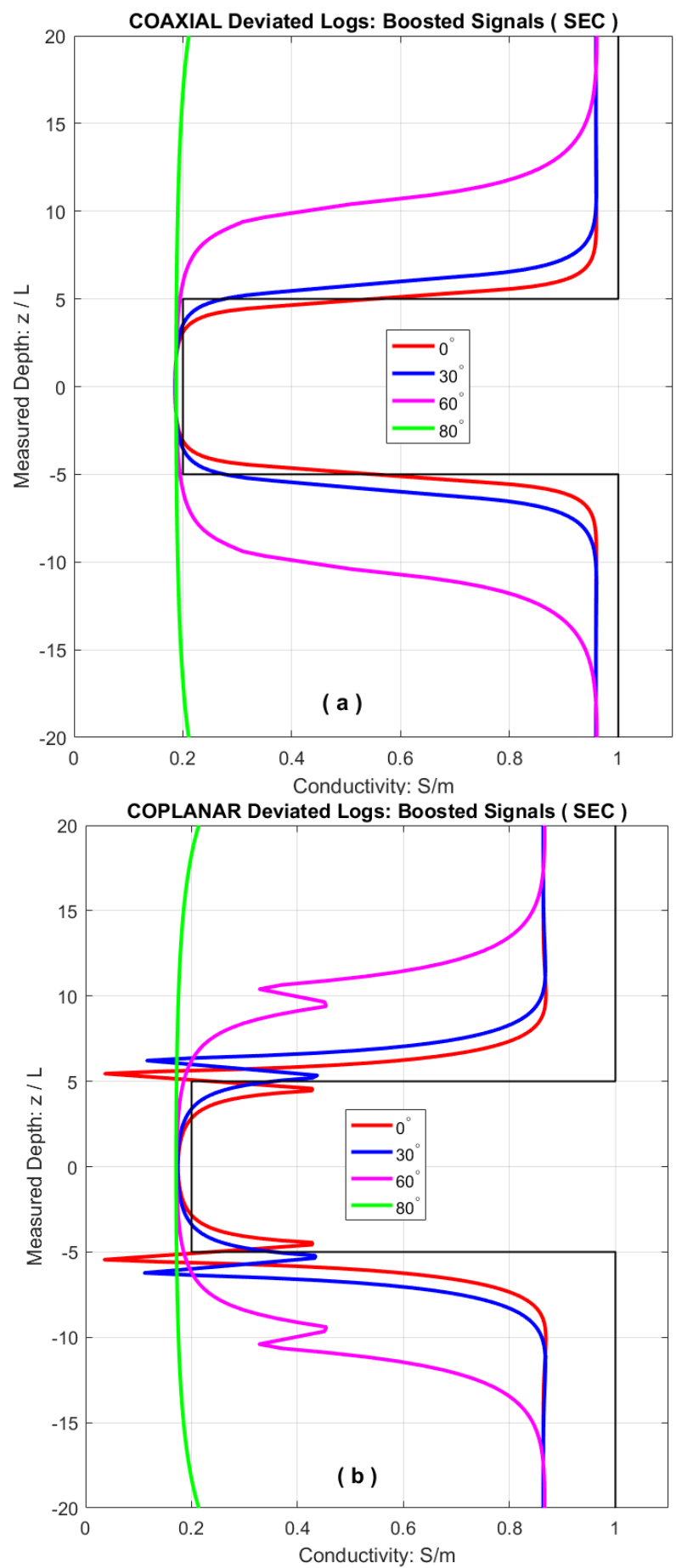

Figure 4 - Coaxial and (b) coplanar boosted signals to a thick bed $(H=10 L)$ crossed by four deviated angles. 

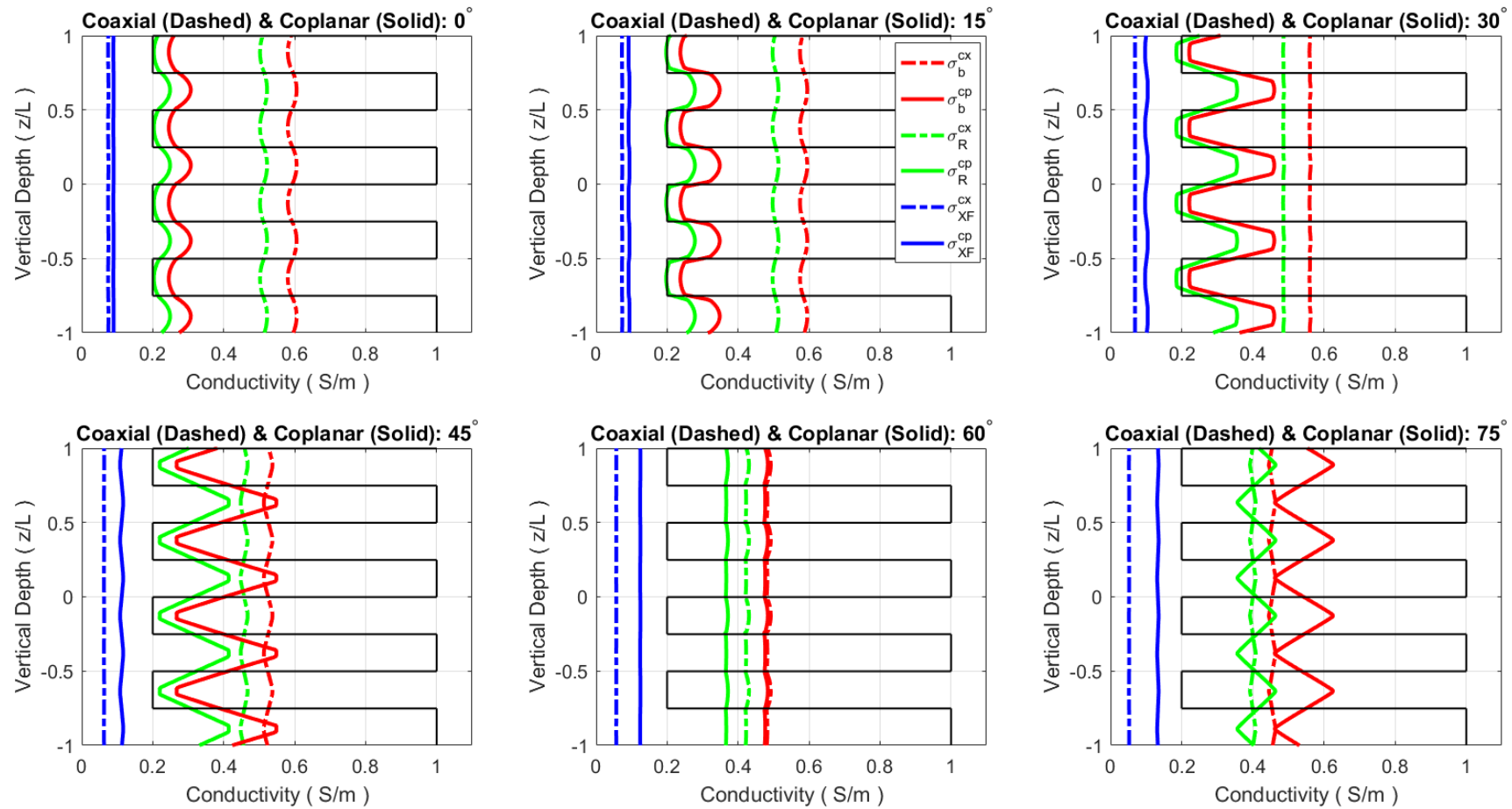

Figure 5 - Effect of the deviated angle on the resistive, reactive and boosted signals of the coaxial and coplanar coil arrays within a thinly $(h=L / 4)$ laminated formation.
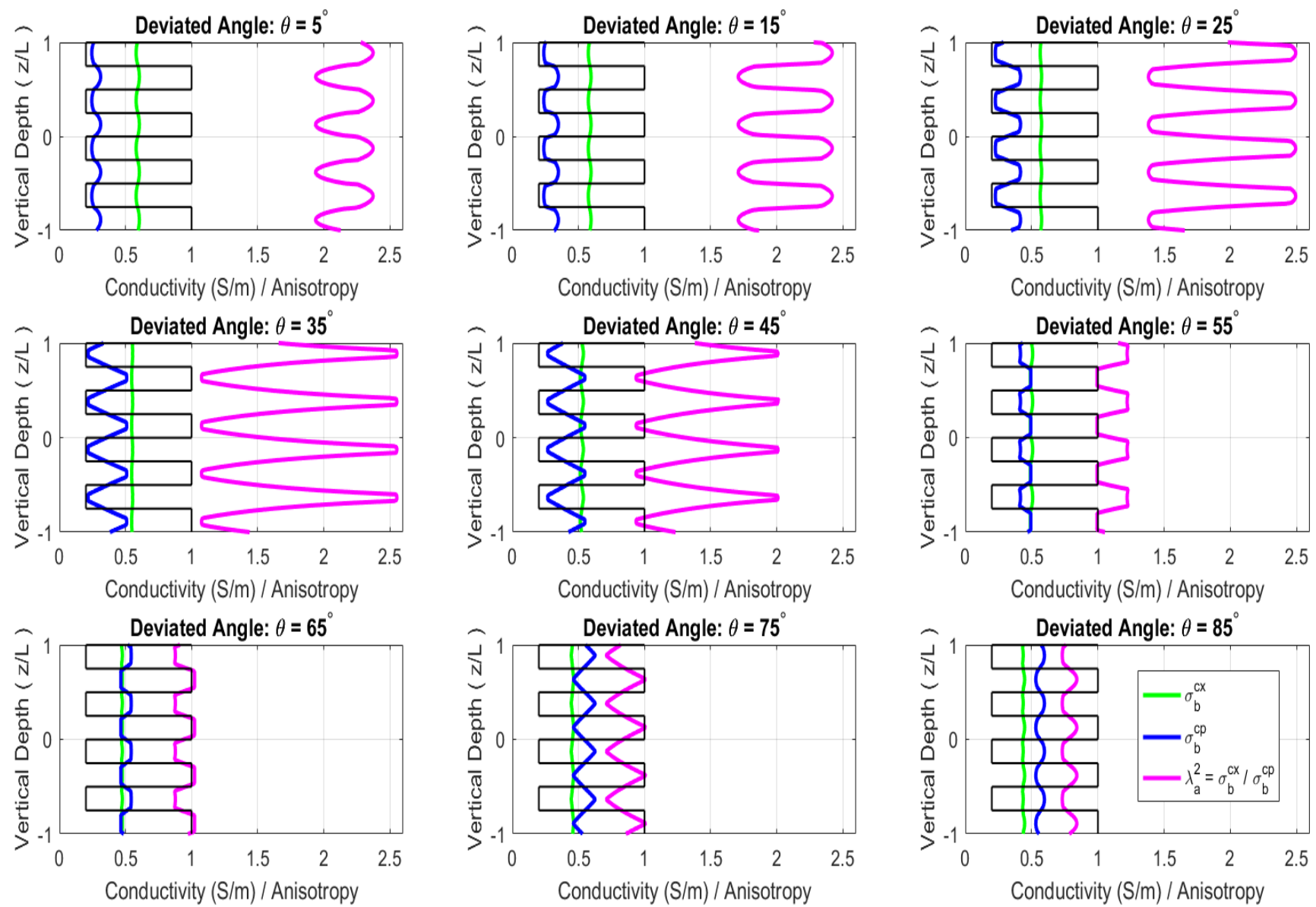

Figure 6 - Effect of the deviated angle on the anisotropy logs obtained by the coaxial and coplanar boosted signals within a thinly $(\mathrm{h}=\mathrm{L} / 4)$ laminated formation. 
These "horns" are more evident on resistive signal. They are caused by the building up of the charges at the boundaries, since the normal component of the electric field is discontinuous at the interfaces.

Now, following Passey et al. (2005), we will define a vertical well as one in which the apparent deviation angle with respect to bedding is less than $30^{\circ}$; a moderately deviated well as one where the apparent deviation angle is between $30^{\circ}$ and $60^{\circ}$; a high angle well as one where the angle is between $60^{\circ}$ and $80^{\circ}$; and a horizontal well as one where the angle is greater than $80^{\circ}$.

We believe that analyzing the angle effect in simple geometries provides basic insight for understanding tool responses in more complex models. Thus, Figure 4 shows the effect of the deviated angles in the coaxial (a) and coplanar (b) boosted signals traversing a thick $(\mathrm{H}=10 \mathrm{~L})$ isotropic bed with symmetrical shoulders. In all logs the bed appears to be thicker at larger dip angles because the vertical axis of the figure denotes the measure depth, not the vertical depth, i.e., more logging points occur inside the bed as the tool passes in ever larger angles. This angle effect can be viewed more clearly in the coplanar logs, since the "double horns" may be a good quality interface indicator. At $60^{\circ}$ the apparent bed thickness (20L) is exactly double the true thickness. As the dip angle increases, the coplanar polarization horns reduced progressively, and the coaxial and coplanar logs tend to become straight lines, since the tool is passing through near the bed center and almost an equal distance to both bed boundaries.

Figure 5 shows the effect of the dip angle on the resistive, reactive and boosted signals of the coaxial (dashed lines) and coplanar (solid lines) arrays within a thick package $(\mathrm{H}=20 \mathrm{~L})$ of a laminated $(\mathrm{h}=\mathrm{L} / 4)$ formation. These logs are right in the middle of the models with the depth ranging from $-2 \mathrm{~L}$ to $2 \mathrm{~L}$, i.e., well away from the shoulder effects. As the dip angle increases, the coaxial logs become increasingly smooth while the coplanar logs show alternating features (smooth and angular) within the laminated formation, analogous to what is shown for vertical logs in Carvalho \& Regis (2016) when they reduced progressively the laminae thicknesses. These feature changes are due to the polarization effects due to the discontinuous electric field at the interfaces. Anderson et al. (1990) show similar features to the coaxial logs to conductivity contrasts and dips above 20 and $60^{\circ}$, respectively. Another effect that increases with the dip angle is that the coaxial and coplanar resistive signals converge, whereas their reactive signals diverge from each other. These polarization effects affect not only the shape (smooth to angular) but also the magnitude of the oscillations, and they cause a curve reversal in some cases, such as at $75^{\circ}$, whereas in other angles there is a perfect correlation with the model.

As we have already described in Equation 11, an apparent anisotropy coefficient can be obtained through the reason between the coaxial and coplanar conductivity logs. Figure 6 shows the effect of the dip angle in the anisotropy logs. As the angle increases they undergo similar feature changes to those in the coplanar logs and their average values reduce progressively so that at $55^{\circ}$ it is already half of the initial value.
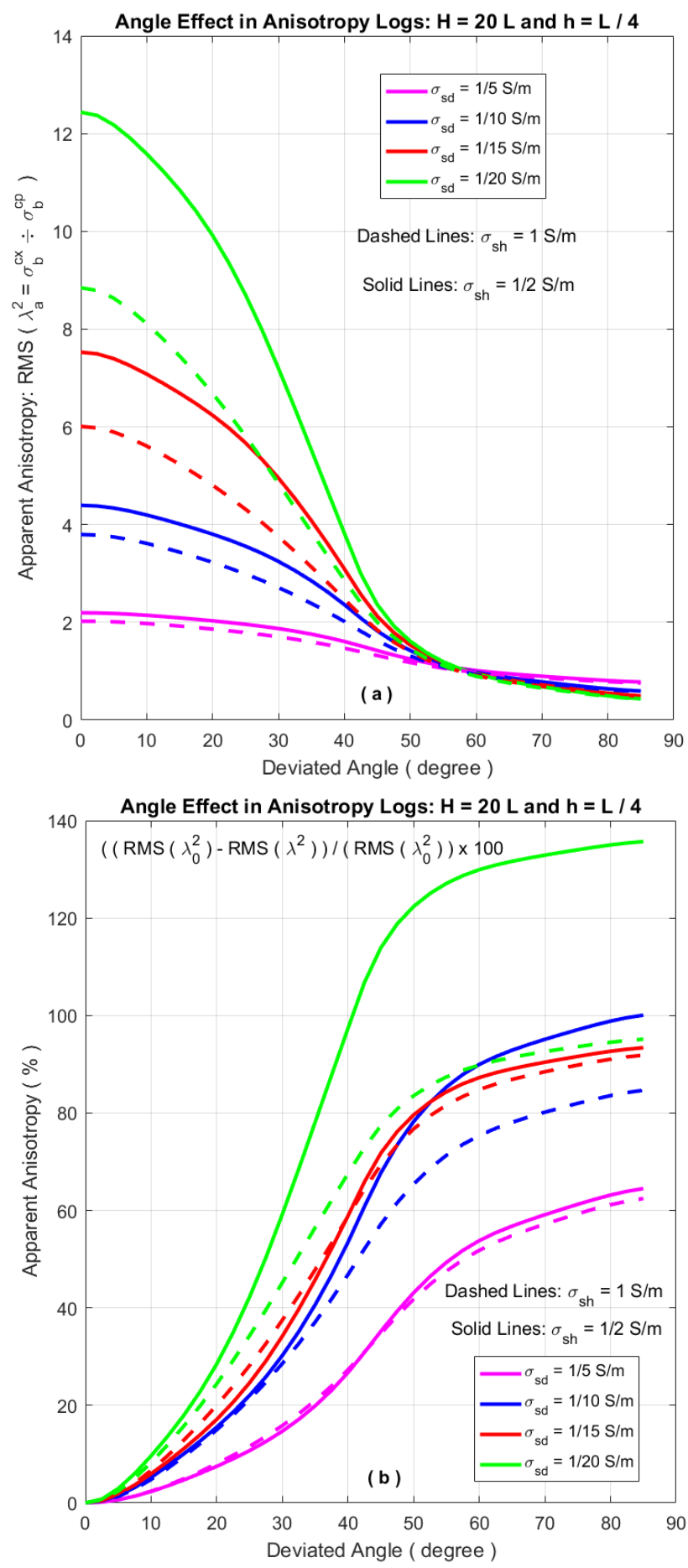

Figure 7 - Absolute (a) and relative (b) effect of the dip angle in the structural anisotropy index within a thinly laminated formation.

Figure 7 shows the absolute (a) and relative (b) variation of the Root Mean Square (RMS) of the apparent anisotropy index within a thinly $(\mathrm{L} / 4)$ laminated formation 
as the deviation angle increases. The analysis of eight different conductivity contrasts (5 to 40 ) between the sand and shale laminae shows that the angle effect increases with the conductivity contrasts.

The deviation loses the technical verticality at $30^{\circ}$. From $0^{\circ}$ to $30^{\circ}$ the apparent anisotropy drops $15 \%$ for a conductivity contrast of 5 , and $60 \%$ for a conductivity contrast of 40 .

Although Anderson et al. (2008) show that when the electrical anisotropy index is higher than five, it alerts the log analyst to look for potential laminated-pay reservoir, we recommend that is imperative to monitor in real time the dip angle of the well and/or the layers, since it strongly affects this anisotropy index and, consequently, finely laminated reservoirs can be underestimated or even ignored.

\section{Conclusion}

In this paper, we showed some numerical responses for one-dimensional (1D) layered anisotropic models, in which the presence of the borehole and the invasion zones are neglected, to simulate geological environments of hydrocarbon reservoirs with structural electrical anisotropy.

The results show that the coaxial and coplanar arrays are very sensitive to the dip angle and so are the apparent anisotropy logs. Thus, when the well is no longer technically vertical, i.e. above 30 degrees, the structural anisotropy estimated by the induction tools decreases significantly, which can lead us to errors in reservoir estimation.

\section{Acknowledgments}

Paulo Carvalho thanks the Cyberspace Institute (ICIBE) of the Federal Rural University of Amazonia (UFRA) for the research support.

\section{References}

ANDERSON B., BARBER T, BASTIA R, CLAUVAUD JB, COFFIN B, DAS M, HAYDEN R, KLIMENTOS T, MINH CC \& WILLIAMS S. 2008. Triaxial induction - A new angle for an old measurement. Oilfied Review.

CLAVAUD JB, NELSON R, GURU UK \& WANG H. 2005. Field example of enhanced hydrocarbon estimation in thinly laminated formation with a triaxial array induction tool: a laminated sand-shale analysis with anisotropic shale. In:Transactions of the SPWLA, paper WW, 46th Annual Logging Symposium, New Orleans.

CARVALHO, de PR \& VERMA, OP. 1999. Coplanar coils response in a borehole. 6th International Congress of the Brazilian Geophysical Society. Rio de Janeiro, RJ, Brazil. http://earthdoc.eage.org/publication/publicationdetails/?pu blication $=47940$
CARVALHO, de P. R \& RÉGIS, C. T. R. 2016. Revisiting some old unexplained effects in induction logs within laminated formations with the triaxial induction tools. Brazilian Journal of Geophysics, v.34, no 3 . http://dx.doi.org/10.22564/rbgf.v34i3.850

CARVALHO, de P. R.; SANTOS, dos W. G. \& RÉGIS, C. T. R. 2010. Fundamentals of coaxial and coplanar coil arrays in induction tools. Brazilian Journal of Geophysics, v.28, no 1 . http://dx.doi.org/10.1590/S0102$\underline{261 \times 2010000100002}$

ELLIS D.V. \& SINGER J.M. 2007. Well logging for earth scientists. $2^{\text {nd }}$ ed. Springer. $692 \mathrm{pp}$.

KAUFMAN, A. A. \& YTSKOVICH G. 2017. Basic Principles of induction logging - Electromagnetic Methods in Borehole Geophysics. Amsterdam Elsevier Publishers. $491 \mathrm{pp}$.

KRIGSHÄUSER B, FANINI O, FORGANG S, ITSKOVICH G, RABINOVICH M, TABAROVSKY L. \& YU L. 2000. A new multicomponent induction logging tool to resolve anisotropic formations: 41st Ann. Logging Sym., Soc. Prof. Well Log. Analysts, paper D.

MORAN J.H. \& KUNZ K.S. 1962. Basic theory of induction logging and application to study of two coil sonds. Geophysics, 27 (6): 829-858.

MORAN J.H. \& GIANZERO S. 1979. Effects of formation anisotropy on resistivity-logging measurements. Geophysics, 44 (7): 1266-1286.

OMERAGIC D., BAYRAKTAR Z., THIEL M., HABASHY, T., ALATRACH S. \& SHRAY F. 2015. Triaxial Induction Interpretation in Horizontal Wells: Mapping Boundaries, Characterizing Anisotropy and Fractures. In: Annual Logging Symposium, 56th. Long Beach, California, USA. Anais SPWLA.

PASSEY Q. R., YIN H. \& RENDEIRO, C. M. 2005: Overview of high-angle and horizontal well formation evaluation: issues, learning, and future directions. 46st Ann. Logging Sym., Soc. Prof. Well Log. Analysts, paper A. 